Revue de droit comparé du travail et de la sécurité sociale

3 | 2017

Le travail dans l'économie informelle, un défi pour le droit social

\title{
La réforme du régime des retraites en Algérie : Un retour à La norme
}

\section{Zina Yacoub}

\section{OpenEdition}

\section{Journals}

Édition électronique

URL : https://journals.openedition.org/rdctss/405

ISSN : 2262-9815

\section{Éditeur}

Centre de droit comparé du travail et de la sécurité sociale

\section{Édition imprimée}

Date de publication : 1 septembre 2017

Pagination : 152-155

ISSN : 2117-4350

\section{Référence électronique}

Zina Yacoub, «La réforme du régime des retraites en Algérie : Un retour à La norme », Revue de droit comparé du travail et de la sécurité sociale [En ligne], 3 | 2017, mis en ligne le 01 novembre 2017, consulté le 04 décembre 2021. URL : http://journals.openedition.org/rdctss/405

\section{(c) $\Theta \Theta \Theta$}

Revue de droit comparé du travail et de la sécurité sociale est mise à disposition selon les termes de la Licence Creative Commons Attribution - Pas d'Utilisation Commerciale - Pas de Modification 4.0 International. 


\section{ZINA YACOUB Université de Béjaia}

\section{LA RÉFORME dU RÉGIME dES RETRAITES EN ALGÉRIE : UN RETOUR À LA NORME}

1 Loi n $16-15$ du 31 décembre 2016 modifiant et complétant la loi $n^{\circ} 83-12$ du 2 juillet 1983 relative à la retraite, JORA $n^{\circ}$ $78 / 2016$.

Voir pour plus de détails : H. Chakib Boukli, « La réforme des retraites en Algérie : loi du 31 décembre 2016 », Revue de Droit Comparé du Travail et de la Sécurité Sociale, n 01/2017, Comptrasec, Bordeaux, pp. 122123.

Voir l'Ordonnance $n^{\circ}$ 97-03 du 11-01-1997, fixant le durée légale du travail, JORA $n^{\circ} 03 \mathrm{du}$ 12-01-1997.

Article 23 de la loi $n^{\circ} 90-$ $11 \mathrm{du}$ 21-04-1990, relative aux relations de travail, modifiée et complétée.
Le régime des retraites en Algérie a connu une réforme aux répercussions non négligeables sur le plan social et financier. La loi 16-15 du 31 décembre 2016 modifiant et complétant la loi 83-12 relative à la retraite ${ }^{1}$, qui est entrée en vigueur le $1^{\text {er }}$ janvier 2017 , entend redresser les dépenses publiques et réinstaurer l'équilibre financier de la Caisse Nationale des Retraites en supprimant le régime de la retraite anticipée, qui avait servi pendant des décennies à maintenir la paix sociale dans une conjoncture économique en transition².

L'article 6 la loi relative aux retraites, dans sa nouvelle version, établit que le travailleur prétendant au bénéfice de la pension de retraite doit obligatoirement réunir les deux conditions suivantes : être âgé de soixante (60) ans, au moins; et avoir travaillé pendant 15 ans au moins. Toutefois, la femme travailleuse peut être admise, à sa demande, à la retraite à partir de l'âge de cinquante-cinq (55) ans révolus. Il est également précisé que pour pouvoir bénéficier de la pension de retraite, le travailleur doit avoir accompli un travail effectif d'une durée au moins égale à sept ans et demi $(7,5)$ et avoir versé l'équivalent en cotisations sociales.

Par ailleurs, le travailleur peut opter volontairement pour la poursuite de son activité, au-delà de l'âge cité, dans la limite de cinq (5) années, au cours desquelles l'employeur ne peut prononcer sa mise à la retraite.

En réalité, ces dispositions ne sont pas très différentes de leurs toutes premières versions, avant que la loi 83-12 ne soit modifiée, d’abord par le décret législatif 94-10 du 26 mai 1994 qui avait institué la retraite anticipée, laquelle pouvait intervenir dans le cadre de compressions d'effectifs, ensuite par l'Ordonnance 97-13 du 31 mai 1997 organisant l'admission à la retraite proportionnelle, et à la retraite sans limite d'âge, sans que cela ne soit lié à des compressions d'effectifs.

Ce faisant, l'ancienne version de l'article 6 a été écartée pour faire place à des dispositions conjoncturelles qui permettaient au salarié :

- soit de bénéficier de la retraite proportionnelle s'il a réuni les deux conditions d'être âgé de 50 ans au moins pour un homme et 45 ans au moins pour une femme, et avoir exercé respectivement un travail effectif durant 20 ans et 15 ans ;

- soit d'être admis à la retraite sans condition d'âge dès lors qu'il avait effectué un travail effectif durant 32 ans. 
La loi 16-15 a donc repris l'essentiel de la loi 83-12 telle que promulguée en 1983, à quelques changements près, notamment en ce qui concerne les dérogations. Il s'agit là d'un retour à la norme, après une gestion conjoncturelle du régime des retraites, dont les conséquences financières ont été jugées excessives par les pouvoirs publics, à un moment où la crise que traverse le pays appelle à réviser la gestion des ressources.

Il convient de noter que certaines dispositions du nouveau texte, sensées modérer l'impact de la réforme sur les catégories de travailleurs les plus vulnérables face à une prolongation de la vie active, sont encore dans l'imprécision et en attente des textes d'application. Il s'agit notamment de l'article 7 qui permet aux travailleurs occupant des postes de travail à haute pénibilité de bénéficier de la pension de retraite avant l'âge prévu, après une durée minimale de travail à ce poste, étant entendu que la liste des postes de travail et les âges correspondants ainsi que la durée minimale passée à ces postes, seront fixés par voie réglementaire.

Pour rappel, cette exception était formulée de façon différente dans l'ancienne version de la loi 83-12, qui prévoyait larticle 7), que les travailleurs occupés dans des emplois présentant des conditions particulières de nuisance, bénéficiaient de la retraite avant l'âge fixé par l'article 6 de la même loi.

Ainsi formulé, le champ d'application de cette exception semble plus retreint que ce qui peut être compris aujourd'hui par emplois à haute pénibilité. Il était prévu que la liste des emplois présentant des conditions particulières de nuisance serait introduite par voie réglementaire. En réalité, cette liste n'a jamais été fixée, ce qui nous conduit à conclure qu'entre 1983 et 1997, les travailleurs exerçant des métiers pénibles n'ont eu aucun moyen juridique de demander la retraite anticipée qui leur revenait, en l'absence des règlements d'application.

Par la suite, et en concomitance avec la légalisation de la retraite anticipée en 1997, la législation sur les relations de travail (loi 90-11, modifiée et complétée), à l'occasion de la réglementation de la durée légale du travail ${ }^{3}$, a renvoyé aux conventions collectives de travail le soin de fixer la liste des travaux particulièrement pénibles et dangereux, impliquant des contraintes sur le plan physique ou nerveux, lesquels ouvrent droit à la réduction de la durée légale du travail ${ }^{4}$. Certes, rien n'indique que cette réduction de la durée du travail puisse impliquer aussi la réduction de l'âge légal pour l'admission à la retraite, mais cette catégorie de travailleurs pouvait bénéficier de la retraite anticipée dans les conditions fixées par l'Ordonnance 97-13 précitée.

Ainsi, tant que la retraite anticipée était ouverte aux travailleurs de tous les secteurs, sans distinction aucune sur la pénibilité, il n'était plus du tout nécessaire de fixer cette liste.

En tout état de cause, la disposition actuelle de l'article 7 a ouvert une brèche dans laquelle se sont introduites plusieurs corporations professionnelles pour affirmer, chacune à son tour, détenir des métiers considérés comme «pénibles ». Ce fut le cas de l'enseignement, de l'agriculture, du transport, de l'industrie, de l'exploitation minière, pétrolière et des collectivités locales. Partant de là, l'Union générale des travailleurs algériens (UGTA) a créé une commission chargée d'élaborer un texte pour déterminer la catégorie des métiers pénibles ${ }^{5}$. Le traitement de cette question ne 
Les retraites anticipée et sans conditions d'âge maintenues pour les métiers pénibles, À la une Actualité Algérie http:// www.liberte-algerie.com/ actualite/les-retraites-anticipeeet-sans-condition-dagemaintenues-pour-les-metierspenibles-249531/print/1. sera pas simple, car rien ne semble interdire une interprétation assez large de la pénibilité pour prendre en compte autant les difficultés morales que physiques.

En attendant l'aboutissement des travaux de cette commission, et en l'absence d'une liste réglementaire antérieure, les pouvoirs publics ont déclaré que les travailleurs exerçant un métier pénible et dangereux continueraient à bénéficier de la retraite sans condition d'âge ${ }^{6}$. Mais puisque la liste de ces travailleurs n'a jamais été fixée par voie réglementaire, il est légitime de se demander ce qui est entendu par métiers pénibles. Faut-il laisser aux travailleurs la liberté d'en formuler la demande, qui sera par la suite examinée et traitée selon les arguments présentés sur la pénibilité du poste occupé ? Ou, faut-il se référer aux clauses conventionnelles qui précisent les éléments composant cette catégorie?

Cette dernière solution peut sembler plus appropriée pour éviter la surcharge des dossiers à traiter. Cependant, l'article 23 de la loi 90-11 prévoit seulement la réduction de la durée du travail pour cette catégorie de travailleurs, et la possibilité de leur faire bénéficier de la retraite sans condition d'âge sous l'égide de l'ancienne législation, en application de l'Ordonnance 97-13, qui s'adressait à tous les travailleurs sans précision, qu'ils exercent des métiers pénibles ou non. Mais avec l'abrogation de cette Ordonnance, il nous semble inapproprié de continuer à appliquer un texte abrogé sur une liste de travailleurs qui n'a jamais été définie. Il y a donc urgence à fixer cette liste pour pouvoir appliquer l'exception de la retraite anticipée aux travailleurs exerçant des emplois pénibles ou dangereux.

A l'opposé, pour les professions hautement qualifiées et les métiers déficitaires en main-d'œuvre, l'âge de la retraite, (article 6 de la nouvelle loi), peut être retardé à la demande du travailleur, (article 7 bis de la même loi), en précisant que la liste des professions hautement qualifiées et des métiers déficitaires ainsi que les conditions et modalités d'ouverture du droit à la prorogation de l'âge de la retraite et aux règles spécifiques de liquidation de la pension y afférentes, seront fixées par voie réglementaire. 
Par ailleurs, la nouvelle loi, larticle 61 bis), prévoit une période transitoire de deux années, durant laquelle le salarié peut bénéficier de la pension de retraite, avec jouissance immédiate, lorsqu'il a accompli une durée de travail effectif ayant donné lieu au versement de cotisations durant au moins trente-deux (32) ans, s'il a atteint ou dépassé, l'âge minimal de cinquante-huit (58) ans en 2017 et cinquante-neuf (59) ans en 2018. Ces dispositions ne peuvent concerner que les travailleurs de sexe masculin, puisque la femme travailleuse, peut déjà, à sa demande, être admise à la retraite à partir de l'âge de 55 ans, et qu'il s'agit d'une règle qui n'est pas limitée dans le temps.

La même disposition légale indique que l'âge prévu à l'article 6 de la loi nº 83-12 du 2 juillet 1983, susvisée, est applicable aux travailleurs cités dans le présent article, à compter

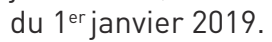

En réalité, cette période de transition, qui donne l'impression que la législation a fait l'objet de deux versions simultanées, trouve sa raison d'être dans le mécontentement de l'opinion publique provoqué par le projet de loi sur les retraites avant même son adoption, ce qui a conduit les pouvoirs publics à intervenir à la hâte pour ajouter l'article 61 bis, dans l'espoir d'atténuer les tensions en permettant à ceux des travailleurs qui auraient 60 ans en 2019, de bénéficier de la retraite anticipée durant ces deux années de transition.

Il faut enfin espérer que ces tensions s'atténuent par la prise de conscience que la réforme du régime des retraites n'est qu'un retour à une gestion normale, et que les conditions exceptionnelles ayant poussé le législateur à légaliser les deux formules de retraite anticipée, font à présent place à d'autres conditions conjoncturelles, marquées par une crise financière alarmante, qui ne peut supporter davantage de déperdition des ressources. 